\title{
A non-authoritative educational metadata ontology for filtering and recommending learning objects
}

\author{
Mimi M. Recker \\ David A. Wiley \\ Department of Instructional Technology \\ Utah State University \\ 2830 Old Main Hill \\ Logan, UT 84332-2830, U.S.A. \\ mimi.recker@usu.edu \\ wiley@cc.usu.edu
}

Paper submitted for publication to the Journal of Interactive Learning Environments: Special issue on metadata

25 September 2000 


\section{Abstract}

Digital libraries populated with learning objects are becoming popular tools in the creation of instructional technologies. Many current efforts to create standard metadata structures that facilitate the discovery and instructional use of learning objects recommend a single, authoritative metadata record per version of the learning object. However, as we argue in this paper, a single metadata record -- particularly one with fields that emphasize knowledge management and technology, while evading instructional issues -- provides information insufficient to support instructional utilization decisions. To put learning objects to instructional use, users must examine the individual objects, forfeiting the supposed benefits of the metadata system. As a solution, we propose a system that includes multi-record, non-authoritative metadata focussed on the surrounding instructional context of learning objects.

Keywords: metadata, learning objects, instructional design, collaborative filtering

\section{Introduction}

The Internet and its application software (e.g., the Web) have become the de-facto resource access and distribution system of the new millennium. However, the Web lacks the standardized structures and typologies found in robust information retrieval systems. Its distributed nature precludes implementing filtering and reviewing conventions typically provided by libraries, reviewers, and publishers. Moreover, a recent study suggests that the coverage of Web content by search engines is continually decreasing, with no more than $16 \%$ coverage by any one engine (Lawrence \& Giles, 1999). At the same time, the study shows that bias in coverage is increasing. 
The full-text approach to searching has also become increasingly ineffective due to the rise in non-textual information online. As a result, the search engine approach generally suffers from low precision and recall.

To address these problems, much recent research has focused on building Internet-based digital libraries, containing vast reserves of information resources. Within educational applications, a primary goal of these libraries is to provide users (including teachers and students) a way to search for and display digital learning resources commonly called 'learning objects'. Examples of such educational digital libraries include www.smete.org, which offers a comprehensive collection of science, math, engineering and technology (SMET) education content and services to learners, educators, and academic policy-makers (Muramatsu, 2000). In Europe, the ARIADNE project has been developing a Europe-wide federation of repositories of multilingual, digital, pedagogical resources (Duval et al., in press).

As part of these efforts, researchers are developing digital library cataloging systems. Much like labels on a can, these labels, or data elements, provide descriptive summaries intended to convey the semantics of the object. Together, the data elements usually comprise what is called a metadata structure (LTSC, 2000). Thus, in typical educational digital library applications, learning objects are stored and labeled with a metadata record. This metadata record usually contains basic information about the object. This may include, for example, technical requirements, rights management, and author demographics. Because of their status as official data descriptors, we call these 'authoritative' metadata. Metadata structures are searchable and thus provide a means for discovering, sharing, and reusing learning objects, even when these objects are non-textual (Duval et al., in press). 
In this paper we examine key assumptions underlying the design of an educational digital library coupled with a metadata structure. In particular, we analyze the fundamental notion that a learning object can be disassociated from its original learning context, effectively described with metadata elements, and then discovered via these descriptions in order to be used or re-used in a new learning context. In short, this paper analyzes the extent to which 'authoritative' metadata support discovery and the instructional reuse of learning objects.

As we explain, our analysis suggests that in addition to 'authoritative' meta-information, a metadata structure must also incorporate what we call 'non-authoritative' metadata. This form of metadata captures the 'embedding' context of a learning object within instruction. For example, these data elements can describe how a learning object was reused, its juxtaposition to other learning objects, and its usefulness in particular instructional contexts. The metadata can also describe the community of users from which the learning object is derived. We argue that this kind of metadata is critical in supporting effective discovery and re-use of learning objects for instructional purposes.

The distinction between authoritative and non-authoritative is primarily based on the differences between the persistent and potentially falsifiable (authoritative) aspects of a learning object (e.g., file size) and the (non-authoritative) context of learning object use and re-use (e.g., its value or usefulness within a particular instructional situation). We wish to specifically address and capture both the former and latter properties in order to support learning object reuse. We also argue that authoritative metadata is generally contributed by the author or authorized catalogers. Non-authoritative metadata, on the other hand, is more likely to be contributed by users of learning objects. 
In particular, we describe and discuss the application of collaborative filtering techniques within a non-authoritative metadata structure in order to support automatic filtering and recommendation of relevant learning objects. We illustrate how a metadata structure based on non-authoritative elements can capture the embedding context of the learning object. We illustrate how it can be comprised of community-contributed information as well as usage metrics. A side benefit of this approach is that it also allows a user to locate other users that share similar interests for further communication, collaboration, and community building.

In the next section of this paper, we discuss fundamental issues surrounding the ontology of metadata structures. We then describe our approach to capturing non-authoritative metadata based upon recent research and techniques in collaborative information filtering. We close with a sketch of an ongoing implementation of these ideas within a system called the Instructional Architect.

\section{The ontology of metadata}

Within education, much recent research has spurred the design and development of digital libraries coupled with metadata. In concert with this work, several groups have focussed on designing metadata structures specifically targeted at describing learning objects. For example, the ARIADNE educational metadata standard is comprised of four categories of metadata: general, technical, semantic, and pedagogic (Duval, et al., in press).

In a related effort, an IEEE standards committee, called the Learning Technologies Standards Committee (LTSC), has developed a draft standard for "Learning Objects Metadata" (LOM). For the purpose of this task, the committee defined a Learning Object as "any entity, digital or non- 
digital, which can be used, re-used or referenced during technology-supported learning" (LTSC, 2000). Note that this is a fairly generic and inclusive definition.

The LTSC learning object model currently defines over 80 tags within its metadata structure. Sample tags include:

Title: $\quad$ The name given to the resource

Language: $\quad$ The language of the intended user of the resource

Description: A textual description of the content of the resource

Interactivity: The type of interactivity supported by the resource

Learning context: The typical type of learners

This learning object metadata structure appears to have wide support in educational technology research communities around the world. In order to support Web integration, researchers have developed an XML (eXtensible Markup Language) binding specification. In addition, the LTSC metadata standard allows for conforming extensions for specific applications.

The LTSC draft standard is designed to provide a means of enhancing the discovery of relevant learning objects. Indeed, the standards are particularly focused on solving the technical aspects of object description and cataloging within a networked environment. They are not, however, focused on capturing aspects surrounding the initial context of instructional use of the object. They do not support encoding a description of the learning activities surrounding a learning object. The standards also do not provide explicit support for the re-use of learning objects within specific instructional contexts. 
In many ways, the efforts to specify authoritative metadata structures raise issues also found the fields of knowledge engineering and knowledge management. Traditionally, knowledge engineering work has focused on building expert systems based on symbolic artificial intelligence (AI) research. Knowledge management systems have focussed on extracting and storing experts' knowledge within a database, often for the purpose of decision support. Despite their promise, such approaches, by and large, have not become widespread. Two key reasons are 1) the knowledge elicitation bottleneck and 2) the difficulty in codifying social context.

In the first case, the design of knowledge systems relies on a description of what experts in the domain know. In practice, it has frequently proven to be very difficult (or very time consuming) for experts to provide a description of their (often tacit) knowledge divorced from the actual activity (Dreyfus, 1993). Removed from the context of their activity, experts have difficulty articulating the skills, knowledge, and heuristics that comprise their expertise.

The second reason is closely related to the first one: in general, designers of knowledge management systems have often failed to focus on the knower. That is, they failed to consider who knew what, why, and in what context. For example, Brown and Duguid (2000) document several cases at large US companies in which attempts were made to codify human knowledge within databases. After the employees with that knowledge left the company, however, they found that the knowledge could not easily be re-created. Much of that 'knowledge' -- in the form of values, contexts, and practice - had left the company with its employees.

Indeed, as argued by research in situated cognition, it may be impossible to separate knowledge from who knows it, and from that person's surrounding community of practice (Brown \& Duguid, 2000; Brown, Collins, \& Duguid, 1989; Suchman, 1987). As demonstrated in many case 
studies, people work and reason within a complex web of practice, skills, spontaneity, and personal relationships. In complex work environments, people constantly shift their goals, preferences, and roles, often due less to knowledge constraints than to subtle power relationships within their operating frame. This background social fabric has proven to be remarkably hard to represent within standard knowledge management systems.

We suspect that attempts to provide an 'authoritative' description of a learning object divorced from context of use raise similar sets of issues. For example, the European ARIADNE project has concluded that it is very difficult to specify a pedagogical metadata set for resources that is applicable across a broad range of cultures (Duval et al., in press). As a result, their pedagogical descriptors are sparse and somewhat generic.

Certainly, some descriptors of learning objects are persistent and canonical, such as a learning object's file size and type. However, many descriptors for learning objects depend critically on the embedding context of the object. Indeed, the activity (for example, instructional design or a learning activity) that led to the representation (the learning object) plays a central role in the creation of that representation. In this way, a learning object is part of a complex web of social relations and values regarding learning and practice. We thus question whether such contextual and fluid notions can be represented and bundled up within one, unchanging metadata record.

Consequently, we argue that for a metadata structure to be effectively support reuse of learning objects within a learning context, it must incorporate what we call non-authoritative records, comprised of non-authoritative data elements. This form of metadata is concerned with describing contextual and changing aspects of a learning object, as opposed to its persistent and non-falsifiable data elements. To support learning object reuse, such non-authoritative elements 
attempt to describe the context of use and surrounding activities of the learning object. They attempt to describe the learning object's worth within a particular learning community (e.g., its usefulness within a particular instructional situation). The data elements can also describe the community of users from which the learning object is derived. Moreover, any user (and not just the authorized cataloger) can contribute a metadata record. As a result, a particular learning resource may have multiple, distributed, non-authoritative metadata records, in addition to an authoritative record. These records may be stored and searched across multiple servers. Thus, the challenge in designing such non-authoritative metadata structures is devising data elements that enable the embedding social fabric of learning object use and reuse within instruction to be captured.

In many ways, the distinction between authoritative and non-authoritative metadata typologies parallels the distinction between what has been called subjective (variable) and objective (factual) types of metadata (Hodgins, 2000). It is also similar to the extrinsic vs. intrinsic distinction made by the Dublin Core (DC) metadata initiative. The DC metadata element set was designed by an international group to support author-generated description of Web resources in order to facilitate discovery (Weibel, 1995). Intrinsic properties are those that are derivable by simply having the resource at hand (e.g., physical form), whereas extrinsic properties describe the context in which the resource is used. The element set was explicitly designed to only concentrate on describing intrinsic properties of resources, and deliberately chose to ignore the description of extrinsic properties.

As we explain in the next section, we believe that capturing and storing such non-authoritative metadata is especially amenable to the application of a recent information filtering technique, called collaborative filtering. In particular, the approach supports discovery and automatic 
filtering and recommendation of relevant learning objects in a way that is sensitive to the needs of particular communities of users interested in teaching and learning. An additional benefit of this approach is that it allows a user to locate other users (students or instructors) that share similar interests for further communication and collaboration.

In the next section of this paper, we describe collaborative information filtering, and briefly discuss its implementation within a system called Altered Vista.

\section{Collaborative information filtering and metadata}

You've arrived in a brand new city, and hunger pangs have erupted. How do you make that all important decision: Where to dine? You might consult restaurant guides, newspapers, or the phone book. More likely, you would ask friends with similar tastes in cuisine to recommend their favorite spots. In the end, you want trusted sources to provide you with information about the quality of restaurants in order to help you make the best selection.

This solution to the 'restaurant problem' is the basic insight underlying research in collaborative information filtering. This approach is based on collecting and propagating word-of-mouth recommendations about the qualities of particular resources (Malone, Grant, Turbak, Brobst, \& Cohen, 1987; Maltz \& Ehrlich, 1995; Shardanand \& Maes, 1995). Systems built on collaborative information filtering approach (often also called recommender systems) have been demonstrated in a variety of domains, including filtering and recommending books, movies, research reports, and Usenet news articles (Resnick \& Varian, 1997).

In ongoing research, we have been applying collaborative filtering techniques within a metadata structure for digital learning resources (Recker, Walker, \& Wiley, 2000). In particular, we are 
developing and evaluating an Internet-accessible system, called Altered Vista, which allows users to share ratings and opinions (a type of non-authoritative metadata) about resources on the Internet. Using the Altered Vista system, users input reviews about the quality and usefulness of Internet-based resources. Their reviews become part of the review (or metadata) database. Users can then access and search the recommendations of other users. Via its recommendation algorithm, the system also supports the automated recommendation of learning resources. In this way, a user is able to use and benefit from the opinions of others in order to locate relevant, quality information, while avoiding less useful sites. Finally, the system can also recommend to a user other users that share similar interests for further communication and collaboration.

In this work, we have adopted an approach where the metadata structure used is specific to the review area (or domain) under consideration. In other words, rather than defining a single, overarching metadata structure for reviewing all learning resources, we define data elements for each review area. These elements then define that metadata structure. Any user can contribute a metadata record using our pre-defined set of data elements for this particular review area. As a result, an individual learning resource may have multiple metadata records. Moreover, a single resource may be reviewed within multiple review areas.

We have been experimenting with a variety of data elements and data types within this system. Some of these involve text entry, while the dimensions of other data elements are represented as 5-point Lickert scales. Example data elements from one implemented review area include:

Quality: $\quad$ The subjective quality of the resource

Educational relevance: The relevance of the resource for education 
Overall rating: The personal overall rating of the resource

The values of these data elements are a function of how individual users perceive them. They are thus fluid, and dependent on the context of use. Typically we have chosen to represent such types as a Lickert scale, allowing users to express a numeric opinion on a scale from 'poor' to 'excellent'.

The ideas underlying non-authoritative metadata apply to the development of learning object metadata structures in several ways. First, by using non-authoritative metadata records, users of objects can contribute information about the embedding context of use of a learning object. For example, users (and not just authors) can describe how they used a learning object, in what types of learning activities they used it, how they juxtaposed it with other learning objects, and how useful they found it.

Second, to maximize the value of this kind of information, we believe it is important to recognize that contributors to the metadata database are members of a community. Information about who contributed is as important as the contribution itself. Hence, users must log in prior to using the system, and their reviews are not stored anonymously. Instead, we believe that the identity of contributing reviewers provides important contextual information within a metadata record. Thus, the author of reviews is both a searchable item and available for inspection within search results. We note that this approach raises privacy issues that we will need to attend to.

Third, as suggested by the research literature, such metadata records can also be analyzed in order to predict how well a user will like unseen objects, given a bank of objects previously rated by a community of users (Konstan, Miller, Maltz, Herlocker, Gordon, \& Riedl, 1997). Thus, 
collaborative filtering algorithms can automatically recommend desirable learning objects to a user. They can also recommend like-minded people (Hill, Stead, Rosenstein, and Furnas, 1995).

Several recommendation algorithms have been developed; they most commonly rely on the nearest-neighbor approach (Herlocker et al., 1999). This algorithm involves selecting a subset of appropriate users based on their similarity to the user desiring recommendations. This subset is called the active user's neighborhood. A common approach for determining neighborhoods is to find a subset of users whose rating values correlate highly: Users with high correlations in their reviews of mutually reviewed objects can be seen as having shared views. After the neighborhood is formed, a weighted aggregate of the neighborhood's ratings for objects unseen by the active user is used to predict ratings for those objects. Objects with predicted ratings above a certain threshold are then recommended to the user.

As previously noted, users within neighborhoods can be seen as sharing similar interests. As such, users within neighborhoods can be automatically recommended to one and another for further communication and collaboration. While much learning object work has been set in a direct instruction paradigm, this kind of community building through learning object rating and use provides a model for learning object reuse within a more open-ended, constructivist learning environment.

Finally, in addition to explicit and actively-contributed non-authoritative metadata, implicit data concerning the use, re-use, and usefulness of a learning object can also be harvested and analyzed. For example, in previous research (Recker and Pitkow, 1996), we showed that access to an online, digital resource is strongly correlated with the recency and frequency of prior usage. 
The collection of such usage metrics is generally easily implemented within a digital library, and thus provides important data concerning object desirability for future users.

Other implicit metrics concerning object desirability include the extent that an object is linked to other objects (Botafogo, Rivlin, \& Shneiderman, 1992; Recker and Pitkow, 1996) and the extent that the content of an object is referenced within other objects (Woodruff, Gossweiler, Pitkow, Chi, \& Card, 2000). The simple inference is that objects that are highly accessed, linked, or referenced are more desirable, and hence should be recommended to users.

These implicit metrics for an object thus also provide a form of non-authoritative metadata. They represent inferences about object desirability, which can be used in ordering search results, and providing recommendations to unseen objects. As such, they provide an important complement to explicitly collected non-authoritative metadata. These implicit measures can also help reduce the cognitive load of users by not requiring them to explicitly enter comments and reviews about each learning object used. These various forms of non-authoritative metadata (user preference, user identity, object usage) can be stored in records alongside the authoritative metadata record. They can then be simultaneously searched to support discovery, recommendation, and re-use of learning objects.

\section{Implementation within the Instructional Architect}

The Instructional Architect is a system that we are currently designing and implementing, which incorporates our ideas based on non-authoritative metadata structures. The system is comprised of a suite of four tools that function together to facilitate the discovery, selection, and instructional use of learning objects stored within a digital library. These four tools are (1) the Discovery Tool, (2) the Presentation, Inspection, and Recommendation Tool, (3) the 
Combination Tool, and (4) the Reflection Tool. Figure 1 shows a sketch of the system, and how the tools interact with digital libraries. For clarity of exposition, major tools are broken into subcomponents. The function of each of these tools and the role of non-authoritative metadata are described below.

\section{Discovery Tool}

The Discovery Tool provides the user a way to initiate a search for one or more learning objects. The tool operates in one of two modes. In simplex mode, the user performs a search against a digital library. An initial test-bed digital library is the NSF-funded SMETE library, Www.smete.org (Muramatsu, 2000), using a simple interface to the library's native search tool. The results from this search are passed across the network to the Discovery tool, which then interprets the results and passes them to the Prediction, Inspection, and Recommendation Tool (PIRT) (described below).

In duplex mode, the user initiates the learning object search from within the Discovery Tool itself. The tool then remotely queries one or more digital libraries, interprets the results, stores them, and passes them to the PIRT. Duplex mode can provide several features not necessarily available in simplex mode, including federated searching across several digital libraries and simultaneous searching against the Instructional Architect's 'non-authoritative' database of collaborative filtering and learning object usage information. This functionality is similar to that of a meta-search engine (e.g., http://www.dogpile.com/ that submits a single query to multiple search engines and organizes the cumulative results for the user. 


\section{Presentation, Inspection, and Recommendation Tool (PIRT)}

The primary function of this tool is to enable users to examine candidate learning objects returned from a search of the digital library. PIRT provides support to users in several ways.

First, it presents the learning objects to the user in a suggested inspection order. That is, when the user searches the digital library for resources, instead of seeing a long list of results in a pseudorandom order, the tool presents the list of candidate learning objects in an order matched to estimated desirability and the user's preferences. PIRT also allows users to preview candidate learning object within the context of the tool.

The algorithm underlying the inspection order is based upon the user's search criteria, user's preferences, and metrics from prior usage of the objects. As previously discussed, these metrics are based on a model derived in our prior research (Recker \& Pitkow, 1996). In particular, we showed that access to an online, digital object is strongly correlated with the recency and frequency of prior object usage.

The user then selects learning objects for further consideration, indicating a preference for objects. The tool uses this selection information to recommend additional learning objects that may be relevant to the user. As previously described, the algorithm underlying these recommendations is based upon research in collaborative information filtering. Specifically, it uses a nearest-neighbor approach to compare information regarding objects previously used together by the user's neighborhood and the group of objects a user has selected in order to recommend additional objects that may be of interest. 
Eventually the user selects some subset of the learning objects returned from both the search and recommender algorithm for utilization. Information about these objects is passed to the Combination Tool.

\section{Combination Tool}

The Combination Tool allows the user to contextualize the learning objects selected in the PIRT. The tool presents the user with the opportunity to sequence the objects as they will appear in the online instruction. For the combined set of learning objects to be more than a digital slide show, they must be contextualized in some manner appropriate to the target learning context. The user can also provide context for the sequenced objects, through the provision of explanatory and transitional text, from within the Combination Tool. The graphic design or screen layout of the objects and surrounding text are controllable through selection of one of a set of user extensible interface templates.

Note that learning objects as defined by the LTSC/LOM working group are generally cases of instructional media. As such, the Combination Tool contains an instructional design coach based on Heinich, Molenda, Russell, and Smaldino's (1999) ASSURE model of instructional media utilization. This coach does not impose an instructional approach or theory upon users; instead it provides optional, Just-In-Time support and guidance to users concerning the effective use of media. A screenshot of the prototype Combination Tool is shown in Figure 2.

The outcome of interaction with the Combination tool is a piece of online instruction, consisting of learning objects and contextualizing information. This instruction can then be downloaded for future use. 


\section{Reflection Tool}

The Reflection Tool is used in a post-hoc manner, having two key functions. First, the tool is used to capture explicit user comments on the effectiveness, ease, and manner in which the various learning objects selected were used. In this manner, the tool can capture the users' context in their use of learning objects. These user recommendations are stored in a nonauthoritative metadata database, along with implicit data concerning the use of learning objects. Using collaborative filtering techniques, such metadata are used to facilitate future recommendation of learning objects from within the PIRT.

Secondly, as previously described, using similar techniques, these non-authoritative metadata records can be used to match people whose usage and recommendations correlate strongly. As these clusters take shape, the Reflection Tool can notify users about their similarities with other members in these emerging communities. Using standard Internet communication tools, users can locate like-minded users to collaborate, communicate, and form new online communities.

\section{Conclusion and discussion}

A single authoritative metadata record can describe a learning object in general (authoritative) terms such as its technical requirements, rights management, and author demographics. However, we argue that records of this type are insufficient to represent the range of information necessary to reuse learning objects in the context of instructional design. Decisions regarding the use and combination of instructional media (which most learning objects qualify as) are decisions about the manner in which to contextualize the media. Therefore, without representing 
information regarding contextualization, metadata cannot fully support the instructional use of learning objects (Wiley, in press).

In this paper, we showed how a particular learning object might have multiple metadata records; these may be referenced within multiple contexts. The customizable metadata structure also enables what we have called non-authoritative data elements to be included. These, we argue, better allow the context of use and re-use of particular learning objects to be described. This supports the discovery of learning objects in a way that is sensitive to the needs of particular communities of users. Moreover, the collaborative filtering approach also supports the automatic recommendation of relevant learning objects. It also allows a user to locate other users that share similar interests for further communication and collaboration.

This paper also presented a partial catalog of the benefits that could be realized through the execution of the approach described. It described a suite of tools that rely upon the approach in order to make such contextual information available to digital library patrons during searching and discovery, while supporting them during the process of instructional design. These capabilities and user services, we believe, are critical to the success of a learning object digital library. 


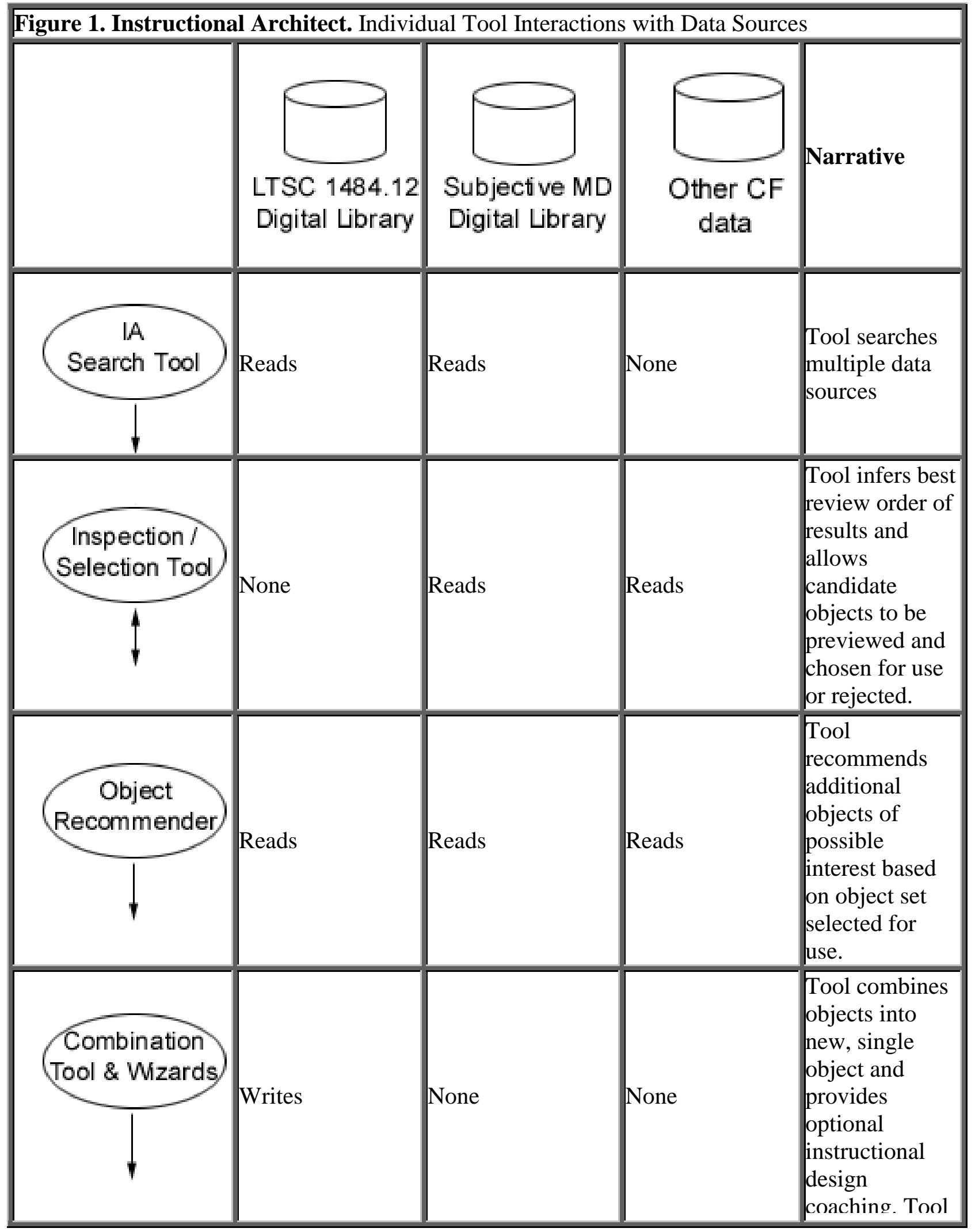




\begin{tabular}{|c|l|l|l|l|}
\hline & & & $\begin{array}{l}\text { adds metadata } \\
\text { for new object } \\
\text { to LTSC DL. }\end{array}$ \\
\hline $\begin{array}{l}\text { Reflection and } \\
\text { Ranking Tool }\end{array}$ & Reads, Writes & Writes & Writes & $\begin{array}{l}\text { Tool captures } \\
\text { context of use } \\
\text { and subjective } \\
\text { user ratings of } \\
\text { component } \\
\text { objects. }\end{array}$ \\
\hline Person & None & & $\begin{array}{l}\text { Tool } \\
\text { recommends } \\
\text { like-minded } \\
\text { people, } \\
\text { supporting } \\
\text { spontaneous } \\
\text { creation of } \\
\text { communities of } \\
\text { interest. }\end{array}$ \\
\hline
\end{tabular}




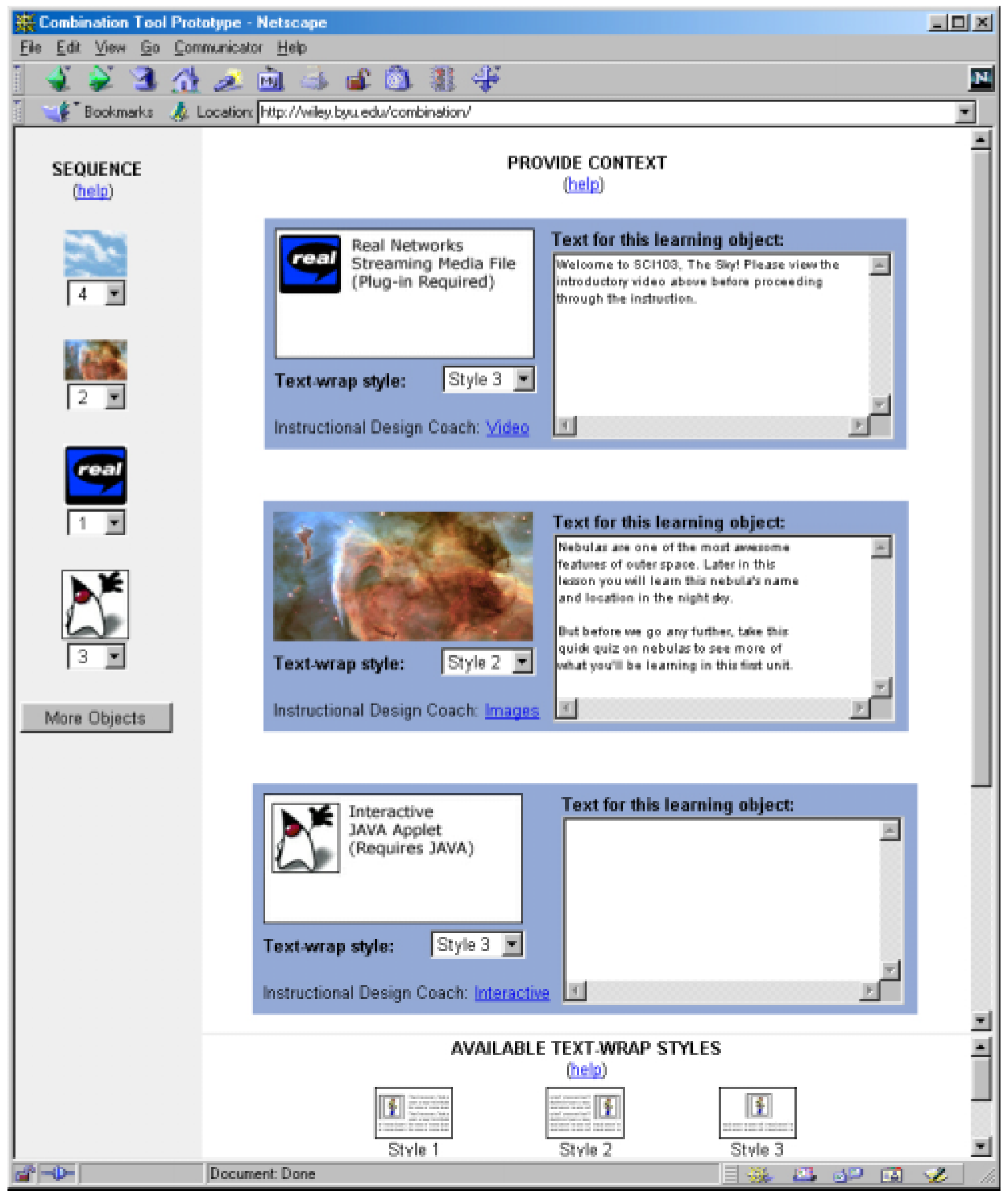

Figure 2. A mock screen shot of the Combination Tool. 


\section{Acknowledgments}

This work reported in this paper was partially supported by a grant from the National Science Foundation (NSF NSDL DUE-0085855) and a Utah State University New Faculty Grant. We thank Andy Walker for his work on the Altered Vista system.

\section{References}

Brown, J.S., Collins, A., \& Duguid, P. (1989). Situated cognition and the culture of learning. Educational Researcher, 18, 32-42.

Brown, J.S., \& Duguid, P. (2000). The social life of information. Cambridge, MA: Harvard Business School Press.

Dreyfus, H. (1993). What Computers Still Can't Do: A Critique of Artificial Reason. Cambridge: MIT Press. (Revised edition of What Computers Can't Do, 1979, 1972.)

Duval, E., Vervaet, E., Verhoeven, B., Hendrikx, K., Cardinaels, K., Olivie, H., Forte, E., Haenni, F., Warkentyne, K., Forte, M., \& Simillion, F. (in press). Managing digital educational resources with the ARIADNE metadata system. Journal of Internet cataloging.

Heinich, R., Molenda, M., Russell, J. D., \& Smaldino, S. E. (1999). Instructional media and technologies for learning, NJ: Prentice Hall. 
Hill, W., Stead, L., Rosenstein, M., \& Furnas, G. (1995). Recommending and evaluating choices in a virtual community of use. In ACM Conference on Human Factors in Computing Systems (pp. 194-201). New York, NY: ACM.

Herlocker, J., Konstan, J., Borchers, A., \& Riedl, J. (1999). An algothmic framework for performing collaborative filtering. In Proceedings of SIGIR'99, pp. 230-237. ACM.

Hodgins, H. W. (2000). Into the future: A vision paper. Commission on Technology and Adult Learning. Retrieved September 20, 2000, from the World Wide Web:

http://www.learnativity.com/download/MP7.PDF

Konstan, J., Miller, B., Maltz, D., Herlocker, J., Gordon, L., \& Riedl, J. (1997). GroupLens. Communications of the ACM, 40(3).

Lawrence, S. \& Giles, L (1999). Accessibility and Distribution of Information on the Web. Nature, 400, 107-109.

LTSC. (2000). IEEE P1484.12 Learning Objects Metadata Working Group homepage [On-line]. Available: http://ltsc.ieee.org/wg12/index.html

Malone, T., Grant, K., Turbak, F., Brobst, S., \& Cohen, M. (1987). Intelligent information sharing systems. Communications of the ACM, 30(5).

Maltz, D. \& Ehrlich, K. (1995). Pointing the way: Active collaborative filtering. ACM Conference on Human Factors in Computing Systems (pp. 202-209). New York, NY: ACM. 
Muramatsu, B. (2000). A Digital Learning Space for Science, Mathematics, Engineering, and Technology Education, Invited Talk, Mathematics/Science Education and Technology 2000 (M/SET), San Diego, CA, February 5, 2000.

Recker, M. \& Pitkow, J. (1996). Predicting document access in large, multimedia repositories. ACM Transactions on Computer-Human Interaction, Vol. 3, No. 4, December, pp. 352-375.

Recker, M., Walker, A., \& Wiley, D. (2000). An interface for collaborative filtering of educational resources. In Proceedings of the 2000 International Conference on Artificial Intelligence (IC-AI'2000): June 26-29, 2000, Las Vegas, Nevada, USA, pp. 317-323.

Resnick, P. \& Varian, H. (Eds.) (1997). Recommender systems, Special Issue. Communications of the ACM, 40(3).

Shardanand, U. \& Maes, P. (1995). Social information filtering: Algorithms for automating word-of-mouth. ACM Conference on Human Factors in Computing Systems (pp. 210-215). New York, NY: ACM.

Suchman, L.A. (1987). Plans and situated actions: The problem of human-machine communication. Cambridge: Cambridge University Press.

Weilbel, S. (1995). Metadata: The foundations of resource description. D-Lib Magazine, July 1995.

Wiley, D. A. (in press). An instructional design theory supporting learning object design and combination. In D. A. Wiley (Ed.), The instructional use of learning objects. Bloomington, IN: Association for Educational Communications and Technology. 
Woodruff, A., Gossweiler, R., Pitkow, J., Chi, E. \& Card, K. (2000). Enhancing a Digital Book with a Reading Recommender. Proceedings of CHI 2000, The Hague, The Netherlands: 153160. 